\title{
Video Article \\ A Real-time Potency Assay for Chimeric Antigen Receptor T Cells Targeting Solid and Hematological Cancer Cells
}

\author{
Biao Xi ${ }^{1}$, Robert Berahovich ${ }^{2}$, Hua Zhou ${ }^{2}$, Shirley $\mathrm{Xu}^{2}$, Yuehua $\mathrm{Wei}^{2}$, Jasper Guan ${ }^{2}$, Hizkia Harto ${ }^{2}$, Jian Guan ${ }^{2}$, Lijun Wu$^{2}$, David Santa Ana ${ }^{1,3}$, \\ Fabio Cerignoil ${ }^{1}$, Brandon Lamarche ${ }^{1}$, Yama A. Abassi ${ }^{1}$, Vita Golubovskaya ${ }^{2}$ \\ ${ }^{1}$ ACEA Biosciences, Inc \\ ${ }^{2}$ ProMab Biotechnologies. Inc \\ ${ }^{3}$ ACEA Therapeutics
}

Correspondence to: Biao Xi at bxi@aceabio.com, Vita Golubovskaya at vita.gol@promab.com

URL: https://www.jove.com/video/59033

DOI: doi:10.3791/59033

Keywords: Immunology and Infection, Issue 153, chimeric antigen receptor, adoptive cell therapy, lentiviral gene transfer, T cells, cancer immunotherapy, RTCA, in vitro, functional assay, cytotoxicity, potency

Date Published: 11/12/2019

Citation: Xi, B., Berahovich, R., Zhou, H., Xu, S., Wei, Y., Guan, J., Harto, H., Guan, J., Wu, L., Santa Ana, D., Cerignoil, F., Lamarche, B., Abassi, Y.A., Golubovskaya, V. A Real-time Potency Assay for Chimeric Antigen Receptor T Cells Targeting Solid and Hematological Cancer Cells. J. Vis. Exp. (153), e59033, doi:10.3791/59033 (2019).

\section{Abstract}

Chimeric antigen receptor (CAR) T-cell therapy for cancer has achieved significant clinical benefit for resistant and refractory hematological malignancies such as childhood acute lymphocytic leukemia. Efforts are currently underway to extend this promising therapy to solid tumors in addition to other hematological cancers. Here, we describe the development and production of potent CAR T cells targeting antigens with unique or preferential expression on solid and liquid tumor cells. The in vitro potency of these CAR T cells is then evaluated in real-time using the highly sensitive impedance-based xCELLigence assay. Specifically, the impact of different costimulatory signaling domains, such as glucocorticoidinduced tumor necrosis factor receptor (TNFR)-related protein (GITR), on the in vitro potency of CAR T cells is examined. This report includes protocols for: generating CAR T cells for preclinical studies using lentiviral gene transduction, expanding CAR T cells, validating CAR expression, and running and analyzing XCELLigence potency assays.

\section{Video Link}

The video component of this article can be found at https://www.jove.com/video/59033/

\section{Introduction}

In recent years, CAR T-cell therapy has been one of the most prominent breakthroughs in cancer immunotherapy for relapsed and refractory hematopoietic malignancies. With the recent U.S. Food and Drug Administration (FDA) approval of CD19-directed CAR T cells for acute lymphoblastic leukemia, non-Hodgkin lymphoma, and diffuse large B-cell lymphoma, and the designation of breakthrough therapy for Bcell maturation antigen (BCMA)-directed CAR T cells for multiple myeloma, this technology has generated great excitement in the scientific community and has fueled numerous basic, applied, and clinical studies worldwide ${ }^{1,2,3,4,5}$. In January of 2019 , more than 700 clinical trials were registered in the clinical trial database (clinicaltrials.gov); about 450 of these trials were either about to start or were actively recruiting patients. Most of the clinical trials are focused on hematological malignancies, and clinical trials utilizing CAR T cells targeting CD20, CD22, and BCMAs, in addition to CD19, are ongoing as well ${ }^{6,7}$. While most of the trials are using autologous CAR T-cell therapy, a significant number of them are also exploring the utility of allogenic CAR T cells ${ }^{8,9,10}$. Despite promising results with hematological malignancies, the use of CAR T cells to target solid tumors has proven to be much more difficult in the clinic for a variety of reasons, including but not limited to the lack of good targets that are exclusively expressed in the tumor, the heterogeneity of solid tumors and tumor "escape", and the difficulty that CAR T cells have in accessing the tumor microenvironment ${ }^{11,12,13,14,15}$. There is a critical need for the development of solid tumor-specific CAR T cells which can overcome these barriers to efficacy and the problem of "on target-off tumor" toxicity. While a multitude of in vitro and in vivo approaches are warranted in the design and testing of CAR T cells, a robust and predictive in vitro potency assay is of primary importance ${ }^{16,17}$.

In order to assess the potency of CAR T cells, various in vitro methods have been developed. In general, these potency assays can be divided into two broad categories depending on whether they (i) directly measure the cytolytic activity of CAR T cells against target tumor cells, or (ii) measure surrogate markers such as cytokines that are released by the CAR T cells as they kill the target cells. Techniques that measure cytolytic activity directly include the chromium-51 release assay (CRA) ${ }^{18}$, imaging-based assays which measure apoptosis of target cells using fluorescent probes ${ }^{19,20}$, and flow cytometry assays that detect apoptotic target cells ${ }^{21}$. In these assays, CAR T cells are typically co-cultured with target cells which have been pre-labeled with radioactive or fluorescent probes, followed by appropriate measurement. Although it has long been considered the gold standard in the field due to its sensitivity, the CRA has some drawbacks. First, it is an endpoint assay and does not provide kinetic information. Second, the target cells need to be labeled with chromium-51 which tends to leach out of the cells and can significantly increase the background noise ${ }^{22}$. Lastly, it requires proper precautions and disposal of the radioactive waste. Alternative assays, which measure byproducts of CAR T-cell interaction with target cells as an indication of potency, include the quantitation of various cytokines released by CAR 
T cells using either flow cytometry-based methods or enzyme-linked immunosorbent assays. Once again, these are endpoint assays which measure the cumulative release of the cytokines at a given time point and, thus, may not necessarily reflect the actual cytolytic activity of the CAR T cells.

When developing a potency assay, particularly one that defines the release criteria for a cell-based therapy such as a CAR T-cell, it is critical that the assay involve minimal manipulations and hands-on time because every interaction is another variable that needs to be accounted for and can diminish the overall robustness and consistency of the assay. Furthermore, the interaction of CAR T cells with the tumor cells is a dynamic process, and providing information about these dynamic interactions, such as the rate of cytolysis, is of primary importance for potency evaluation. With these criteria in mind, we developed a label-free kinetic potency assay for CAR T cells that utilizes the XCELLigence realtime cell analysis (RTCA) platform. xCELLigence utilizes specialized microtiter plates (E-Plates) that contain gold biosensors embedded in the bottom of each well. Working with either adherent solid tumor cells, or liquid cancer cells that have been tethered using specific antibodies, these biosensors monitor in real-time CAR T cell-induced changes in target cell number, cell size, cell-substrate attachment strength, and cell-cell interactions (i.e. barrier function) $17,23,24,25,26,27,28$. The workflow is simple and involves simply seeding the target cells into the wells of E-Plates, followed by the addition of the CAR T cells at different effector-to-target ratios (Figure 1). Subsequently, as the biosensors continuously monitor the viability of the target cells the data is automatically displayed in real-time.

Over the past 15 years the xCELLigence assay has been validated for assessing the potency of natural killer (NK) cells, T cells, CAR T cells, checkpoint inhibitors, bispecific antibodies, oncolytic viruses, and some combination therapies ${ }^{17,29,30,31,32,33,34}$. Recently, the $\times C E L L i g e n c e$ potency assay was evaluated for manufacturing T-cell receptor (TCR)-engineered T-cells ${ }^{35}$. Here we report employing the RTCA system for evaluating the in vitro potency of CAR T cells designed to target solid tumors and liquid tumors in clinical therapies.

\section{Protocol}

\section{Generation of CAR-encoding lentivirus}

NOTE: Once the specific CAR T-cell plasmid construction is completed (CD47 and others), lentiviral CARs are generated by the standard procedure, using 293 FT cells, a lentiviral packaging mix, and transfection agents (see the Table of Materials) as described ${ }^{29}$. Subsequently, use a quantitative reverse transcription polymerase chain reaction (RT-PCR) kit and a thermal cycler (see the Table of Materials) to determine the virus titer by measuring the lentiviral RNA amount according to the manufacturer's protocol. It is important that all lentiviral procedures be carried out strictly following safety requirements.

1. Seed $15 \times 10^{6}$ HEK293FT cells in Dulbecco's modified Eagle's medium (DMEM) and incubate the cells overnight at $37^{\circ} \mathrm{C}$ in one $150 \mathrm{~mm}$ dish inside a humidified $5 \% \mathrm{CO}_{2}$ incubator.

2. Prepare two $15 \mathrm{~mL}$ tubes with transfection complex. The first tube contains lentiviral vector plasmid DNA ( $5 \mu \mathrm{g})$ and lentiviral packaging mix $(22.5 \mu \mathrm{g})$ in $2.5 \mathrm{~mL}$ of transfection dilution solution. The second tube contains $82.5 \mu \mathrm{L}$ of transfection reagent in $2.5 \mathrm{~mL}$ of transfection dilution solution (see the Table of Materials).

3. Pipet the contents of tube 1 into tube 2, and incubate the mixture at room temperature for $15 \mathrm{~min}$.

4. Transfer the contents of the tube dropwise to the dish of HEK293FT cells and incubate the sample overnight at $37{ }^{\circ} \mathrm{C}$ in a humidified $5 \% \mathrm{CO}_{2}$ incubator.

5. The next day, replace the existing medium with $19 \mathrm{~mL}$ of fresh DMEM culture medium and continue to incubate the cells overnight inside the humidified $5 \% \mathrm{CO}_{2}$ incubator at $37^{\circ} \mathrm{C}$.

6. Transfer the medium from the dish to a $50 \mathrm{~mL}$ centrifuge tube. Keep the tube with the virus-containing medium in the refrigerator.

7. Repeat the above procedure, adding fresh DMEM and collecting it again after 1 day.

8. Combine two collections of the media into one centrifuge tube. Centrifugate the tube at $2,000 \times g$ for 30 min at $4{ }^{\circ} \mathrm{C}$.

9. Transfer most of the lentivirus-containing supernatant to an ultraclear centrifuge tube. Leave a minimum volume, about $1 \mathrm{~mL}$, of the supernatant to avoid disturbing the pellet which may contain cells and/or debris.

10. Ultracentrifuge the above clarified supernatant at $110,000 \times \mathrm{g}$ for $100 \mathrm{~min}$ at $4{ }^{\circ} \mathrm{C}$.

11. Remove the supernatant carefully and gently add $100 \mu \mathrm{L}$ of DMEM medium to the virus pellet at the tube bottom. Leave the tube on ice for 15 $\min$. Mix the solution gently and aliquot the lentivirus solution into prechilled sterile tubes. Store these virus stock tubes in a $-80^{\circ} \mathrm{C}$ freezer.

12. Use a quantitative RT-PCR kit to determine the titer of the lentivirus according to the manufacturer's protocol, which extracts and measures lentiviral RNA.

\section{Generation and expansion of CAR T cells}

1. Activate previously frozen human PBMCs (about $1 \times 10^{6}$ to $2 \times 10^{6}$ cells) in $1 \mathrm{~mL}$ of CAR T-cell medium with an equal number of CD3/CD28coated microbeads (see the Table of Materials) and incubate the cells at $37^{\circ} \mathrm{C}$ in a humidified $5 \% \mathrm{CO}_{2}$ incubator for $24 \mathrm{~h}$.

2. Thaw an aliquot of the lentivirus stock on ice.

3. Add $1 \mu \mathrm{L}$ of transduction enhance agent into the well with the cells and mix.

4. Add lentivirus to the cells at a multiplicity of infection (MOI) of 5:1 and mix gently. On the next day, repeat this step ( $24 \mathrm{~h}$ after the first transduction).

5. Monitor the T cell growth every 2-3 days. Add more fresh CAR T-cell medium to maintain the cells at a density of $1 \times 10^{6}$ to $2 \times 10^{6}$ cells $/ \mathrm{mL}$.

6. Freeze down the CAR T cells with a standard protocol using freezing solution (see the Table of Materials).

7. Thaw CAR T cells using a standard method and preculture them in the CAR T-cell medium for about $\sim 2-4 \mathrm{~h}$ with IL-2 (300 units/mL) before applying them to the assay. 


\section{Detection of CAR expression by flow cytometry}

1. Transfer $3 \times 10^{5}$ CAR T cells and nontransduced T cells to two separate $1.5 \mathrm{~mL}$ microcentrifuge tubes.

2. Centrifuge the tubes at $300 \times g$ for 2 min and resuspend the cells in $200 \mu \mathrm{L}$ of fluorescence-activated cell sorting (FACS) buffer containing $1 \%$ human serum.

3. Pipet $100 \mu \mathrm{L}$ of cell solution into two $5 \mathrm{~mL}$ polystyrene FACS tubes and keep the tubes on ice for $5 \mathrm{~min}$.

4. Add $1 \mu \mathrm{L}$ of biotinylated goat anti-mouse $\left.\mathrm{F}(\mathrm{ab})_{2}\right)_{2}$ to one tube of each cell type. Then, add $2 \mu \mathrm{L}$ of PE-labeled anti-tag antibody (see the Table of Materials) to the other tube of each cell type. Mix well and incubate them on ice for $30 \mathrm{~min}$.

5. Wash the cells with $3 \mathrm{~mL}$ of FACS buffer in each tube and centrifuge the tubes at $300 \times g$ for 5 min; discard the supernatants and vortex very briefly or shake the tubes briefly to resuspend the cells in the residual liquid.

6. Add $2 \mu \mathrm{L}$ of APC anti-CD3 and $2 \mu \mathrm{L}$ of 7-AAD antibody solution (see the Table of Materials) to each tube. In the tube of cells stained with anti- $\mathrm{F}\left(\mathrm{ab} \mathrm{b}_{2} \mathrm{Ab}\right.$, add $1 \mu \mathrm{L}$ of PE-labeled streptavidin. Mix briefly and incubate the tubes on ice for 30 more min.

7. Use FACS buffer to wash the cells again as described in step 3.5 and add an additional $200 \mu \mathrm{L}$ of FACS buffer to each tube.

8. Use flow cytometry to analyze the cells by first gating on the T cells in a forward scatter vs. side scatter plot and then gating on the live cells (7-AAD-negative) in a CD3 vs. 7-AAD plot. The final step is to analyze anti-tag, anti-ScFv or anti-F(ab') ${ }_{2}$ vs. CD3.

\section{Real-time cytolysis potency assay}

NOTE: Perform the RTCA assay according to the manufacturer's recommended conditions. In brief, first plate the target cells in the wells of the E-Plate, followed by the addition of CAR T cells on the next day. The cytolysis activity of CAR T cells against target cells is monitored in realtime. T cells and mock-transduced T cells (Mock CAR T cells) are used as negative effector cell controls. The following protocol describes an in vitro real-time cytolysis potency assay for adherent tumor cell lines.

1. Add $100 \mu \mathrm{L}$ of target cell culture medium to each well of the $x$ CELLigence E-Plate, place the plate inside the xCELLigence instrument, and take a background reading. Then, transfer the E-Plate to a tissue culture hood for cell seeding.

2. Use a standard protocol to trypsinize target cancer cells from the culture device. Then transfer the cells to a $15 \mathrm{~mL}$ centrifuge tube and add fresh culture medium, up to $15 \mathrm{~mL}$. Pellet the cells by centrifugation for $5 \mathrm{~min}$ at $200 \mathrm{xg}$. Discard the supernatant, add $5 \mathrm{~mL}$ of fresh medium, and use a serological pipette to gently resuspend the cell pellet. Count the density of live cells using a hemocytometer and microscope.

3. Adjust the cell density appropriately and then add $100 \mathrm{~L}$ of the cell suspension to each well of the E-Plate. The target cell number is typically around 10,000 cells/well for adherent cell lines (BxPC3, Hela-CD19, and SKOV3), or 30,000 cells/well for suspension cells such as Raji (see below for details regarding precoating wells with antibodies in order to tether liquid cancer cells).

4. Equilibrate the E-Plate at room temperature for $30 \mathrm{~min}$ to allow the cells to settle evenly on the bottom of the well (this is critical; do not skip this step).

5. Place the E-Plate into the XCELLigence instrument inside the cell culture incubator and start to measure impedance, displayed as Cell Index vs. time, automatically every $15 \mathrm{~min}$.

6. The next day, prepare effector CAR T cells. Make sure to prepare appropriate controls (i.e., Mock CAR T cells, non-transduced control T cells, and/or unrelated CAR T cells) ahead of time to ensure all the cells are ready at the same time. Adjust the effector cells and the control cells to the proper density, and prepare serial dilutions to ensure the desired E:T ratios will be achieved when $100 \mu \mathrm{L}$ of effector cell suspension is added to each well in step 9.

7. Pause $X C E L L i g e n c e$ data acquisition and bring the E-Plate from the incubator to a cell culture hood.

8. Remove $100 \mu \mathrm{L}$ of medium from each well. The amount of residual medium in each well is now $100 \mu \mathrm{L}$.

9. Add $100 \mu \mathrm{L}$ of serially diluted effector CAR T cells or other control cells (i.e. Mock CAR T cells) to achieve the desired E:T ratios.

10. Equilibrate the E-Plate at room temperature for $30 \mathrm{~min}$ to allow effector cells to settle, and then place the E-Plate back to into the xCELLigence instrument. Resume data acquisition.

11. If so desired, at key time points $x$ CELLigence data acquisition can be paused and the plate removed in order to collect small samples to be analyzed by orthogonal assays (i.e., measuring cytokine production by ELISA or flow cytometry).

12. In the EGFR-GITR-CD3 CAR T-cell experiment, measure INFY yield with an ELISA kit (follow the instructions of the manufacturer; see the Table of Materials).

NOTE REGARDING THE USE OF LIQUID CANCERS: For testing nonadherent hematological cancer cells, prior to adding cells the wells of the E-Plate are first coated with an antibody that is specific for an antigen that is expressed on the surface of the cancer cells. For the Raji $B$ cell line a tethering reagent based on anti-CD40 is used (see liquid tumor killing assay kit in the Table of Materials). Below is the procedure for coating the plate:

13. Dilute the tethering reagent (anti-CD40) with tethering buffer to a concentration of $4 \mu \mathrm{g} / \mathrm{mL}$.

14. Working inside a tissue culture hood, add $50 \mu \mathrm{L}$ of the diluted tethering reagent to each well of the E-Plate. Leave the E-Plate at room temperature or in a $37^{\circ} \mathrm{C}$ incubator for $3 \mathrm{~h}$.

15. Remove the tethering reagent and wash the E-Plate at least $2 x$ with wash buffer. At this point, the E-Plate is ready for seeding the Raji target cells $(30,000$ cells/well).

16. Continue with step 4.1 (above) to perform the rest of the procedure. 


\section{Representative Results}

\section{CAR lentivirus preparation and CAR T-cell generation and potency assessment}

The titers of the CAR lentivirus preparations were determined using a quantitative RT-PCR kit (see the Table of Materials) according to the manufacturer's protocol. The titration protocol extracted virus RNA first and then measured the lentiviral RNA copy number, which indicated the amount of infectious viral particles. The titer of virus generated from one $150 \mathrm{~mm}$ dish using the above protocol usually ranges between $10^{9}-10^{10}$ viral copies $/ \mathrm{mL}$. Figure 2A shows the RT-PCR cycle number vs. the signal strength from a representative quantitative PCR result. Once the virus quality was satisfied, when the titer was larger than $1 \times 10^{8} \mathrm{pfu} / \mathrm{mL}$, it was frozen down for subsequent T cell transduction. After the CAR T cells were transduced with lentivirus, the T cells were cultured for an additional $12-14$ days, maintaining their density around $1 \times 10^{6}$ to $2 \times 10^{6}$ cells $/ \mathrm{mL}$. The CAR T cells were then checked with anti-ScFv-specific antibody using a flow cytometer before a downstream application or freeze down. A good representative batch result is shown in Figure 2B. Using an anti-ScFv antibody, about 50\% of the CAR T cells stained positive (Q2 $50.6 \%$ vs. T cells $1 \%$ ), indicating the expression of CAR in about $50 \%$ of the T cells. Subsequently, the RTCA potency assay was performed for cytolysis determination before each batch of CAR T cells was frozen down and ready for future application. One cycle of the CAR T-cell design, generation, and assessment procedure took about 1 month.

\section{Killing of Raji lymphoma B cells by CD22-CAR T cells}

The anti-CD40 liquid tumor tethering kit (see the Table of Materials) was used at a concentration of $4 \mu \mathrm{g} / \mathrm{mL}$ to coat an E-Plate for $3 \mathrm{~h}$ at $37{ }^{\circ} \mathrm{C}$. After washing the wells with tethering buffer, B-cell lymphoma cells were added to the E-Plate at a density of 30,000 cells/well. After allowing the cells to settle for $30 \mathrm{~min}$ the E-Plate was placed back inside the xCELLigence instrument, and impedance readings were immediately initiated in order to capture cell attachment and proliferation. The following day, either CD22-CAR T cells, Mock CAR T cells, or untransduced T cells were added. In Figure 3, an E:T ratio of 10:1 was used for all cell types. CD22-positive Raji cells, treated with CD22-CAR T cells displayed significant killing (green trace) compared to the negative controls (untransduced T cells and Mock CAR T cells).

\section{Effective killing of pancreatic cancer cells by CD47-CAR T cells}

CD47 is a transmembrane surface glycoprotein of the immunoglobulin superfamily. As an integrin-associated protein, it is highly expressed in both hematological cancers (leukemia, lymphoma, and multiple myeloma) and solid cancers (such as ovarian, small cell lung cancer, pancreatic, glioblastoma) and other types of cancers ${ }^{36,37}$. CD47 is also known as a do-not-eat-me signal to macrophages, which has made it a potential therapeutic target in some cancers. CD47-CAR T cells were produced and tested against BxPC3 pancreatic cancer cells which express highlevels of CD47 $7^{38,39}$. BxPC3 cells were seeded in the E-Plate on day 1 at a density of 10,000 cells/well. Real-time monitoring showed that these cells reached confluency after $16 \mathrm{~h}$. At this time point, CAR T cells were added at the E:T ratio of 10:1 (Figure 4A). Control effector cells like nontransduced T cells and Mock CAR T cells were also added. The results clearly show that CD47-CAR T cells selectively kill the target BxPC3 cells $^{29}$. Also, Figure 4B shows that the impedance signal when CD47-CAR T cells are added to an empty well is substantially lower than the impedance signal generated by target BXPC3 cells. The Cell Index value from wells with CD47-CAR T cells alone reached a maximum of 0.14 , which is only slightly higher than the signal from medium alone (0.02). This indicates that in this heterogeneous killing assay, the impedance signal is derived almost exclusively from the target cancer cells.

\section{Costimulation of CAR T cells by the GITR domain}

When expressed in an EGFR-GITR-CD3 CAR, the GITR costimulatory domain was previously reported to enhance the killing of EGFR-positive SKOV3 cancer cells but not EGFR-negative MCF-7 cancer cells ${ }^{30}$. To better clarify the role of the GITR domain, CAR constructs containing the full-length GITR domain, or deleted or rearranged versions of the domain, were generated and tested for the ability to coactivate CAR T cells (Figure 5A). The XCELLigence data in Figure 5B,C shows that the GITR costimulatory domain enhances CAR T cell killing of EGFR-positive target cells above what is observed with the original CAR construct which lacks the GITR domain. Furthermore, deleting 10 amino acids from the GITR domain (amino acids 184-193) abolished this stimulatory activity. In contrast, rearranging the relative positioning of the GITR domain within the CAR construct proved to be less detrimental to its stimulatory capabilities. Using end point data, IFNy production as a surrogate of Tcell activation also demonstrated the stimulatory activity of the GITR domain (Figure 5B). In contrast to end point data which is a mere "snap shot", the continuous impedance profile of the XCELLigence instrument clearly illuminates subtle differences in the killing kinetics of the different treatments (Figure 5C). The results obtained here are consistent with those of an in vivo study ${ }^{30}$. 


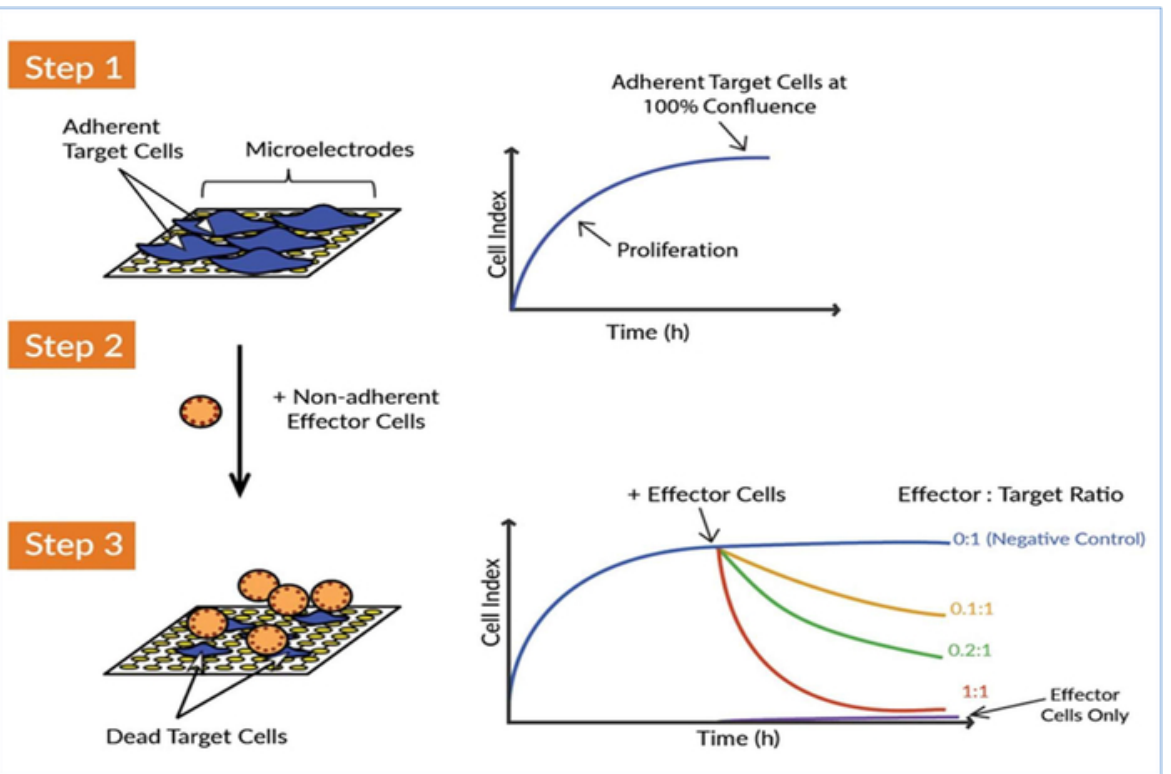

Figure 1: The xCELLigence real-time cell analysis (RTCA) system detects the killing of target cells by effector cells. There are three main conceptional steps to measuring the killing activity of CAR T effector cells against target cancer cells. Step 1: Seed the target cells (i.e., tumor cells) into the well of an E-Plate. The cells attach to the gold biosensor microelectrodes and this impedes the flow of electric current between the electrodes. This impedance value is measured and plotted as a unitless parameter called Cell Index. The Cell Index value increases as the cells grow and reaches a plateau as the cells approach confluence. Step 2: Effector cells-nonadherent immune cells-are subsequently added. Because these cells do not adhere to the gold microelectrodes, they do not directly cause an impedance change. Step 3: If the effector cells attack the target cancer cells, the destruction of the tumor cells is reflected by a decrease in Cell Index over time. This cytolytic activity of the effector cells, or the potency of the effector cells, can be sensitively and precisely monitored. The continuous acquisition of impedance data from the XCELLigence system enables real-time killing kinetic analysis for multiple conditions simultaneously. Please click here to view a larger version of this figure.

A

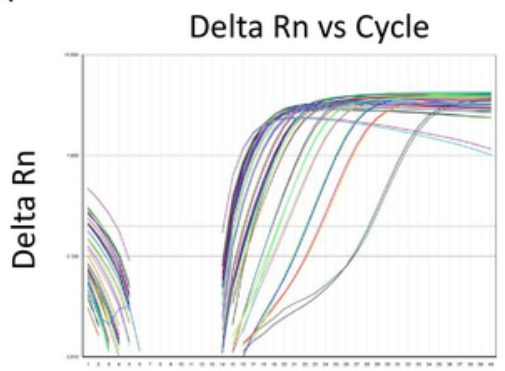

Cycle Number
B

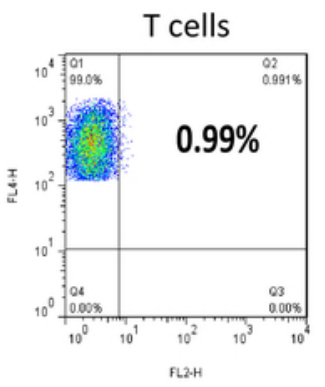

CD19-CAR-T Cells

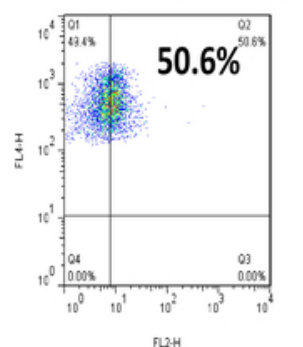

Fuㅐ

Rabbit CD19 ScFv(FMC63) Ab

Figure 2: Titer determination of the lentivirus and flow cytometry evaluation of the CAR T cells. (A) Lentiviral titer determination. The different color lines are representative samples for cycle number vs. delta Rn. Low cycle numbers indicate a relatively high amount of virus RNA template present in the samples. (B) After transduction, CAR T cells were cultured and maintained at a density of less than $2 \times 10^{6}$ cells $/ \mathrm{mL}^{2}$ and then subjected to flow analysis. The $y$-axis reflects staining for T cells, with positive values reflected in areas Q1 and Q2. Both samples are $100 \%$ positive for T cells. The expression of CAR scFV is determined using an scFv-specific Ab ( $x$-axis). The result shows that more than $50 \%$ of the T cells are scFv positive in CD22-CAR T cells. Please click here to view a larger version of this figure. 


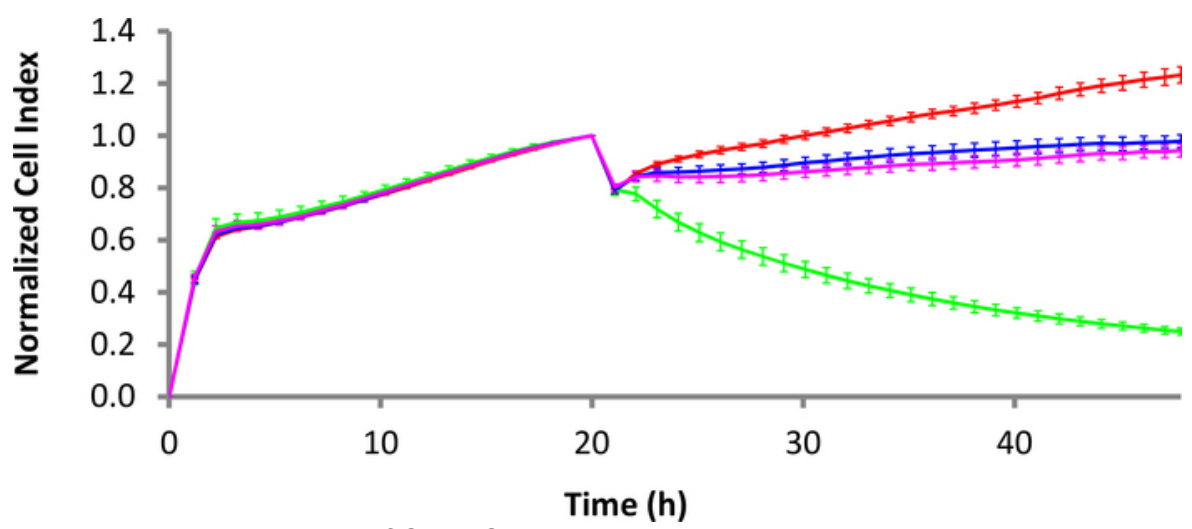

Figure 3: Killing dynamics of CD22-CAR T cells against Raji Burkitt's lymphoma cells. While the red curve is the Raji cells alone, the green curve is the Raji cells treated with CD22-CAR T cells. The pink and blue curves are Mock CAR T-cell treatment and nontransduced T-cell treatment, respectively. The E:T ratio is 10:1. Error bars are standard deviation. The time scale is set up at $2 \mathrm{~h}$ intervals for easy display although more data points are available. Please click here to view a larger version of this figure.

A
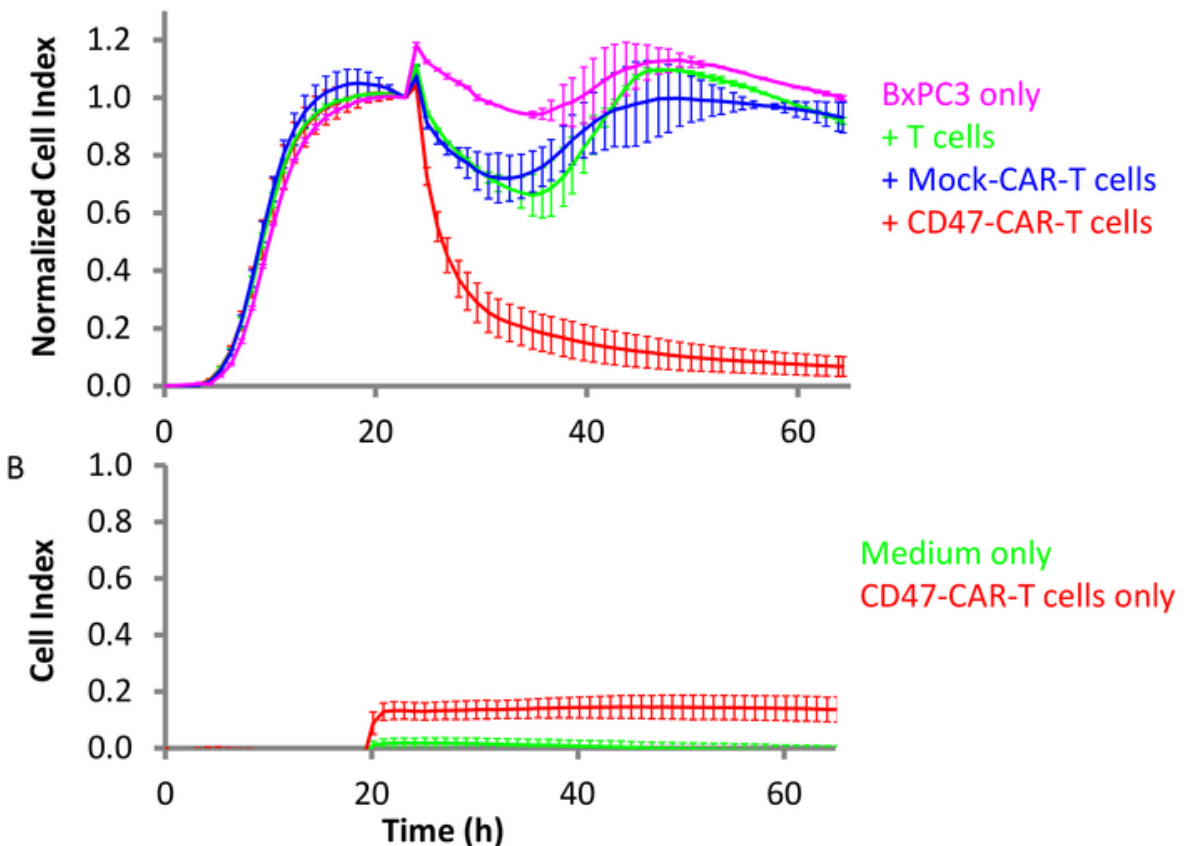

Figure 4: Efficacy of CD47-CAR T cells against pancreatic solid tumor cells. (A) Pink is the target BxPC3 cells alone, while red is BxPC3 with CD47-CAR T cells added. Green is BxPC3 with the addition of nontransduced T cells, and blue is with Mock CAR T cells. The E:T ratio is 10:1. (B) In the same setting, red is empty (lacking target cells) control wells with CD47-CAR T cells only and green is the medium only. Error bars are standard deviation. Please click here to view a larger version of this figure. 
A

\begin{tabular}{|c|c|c|c|c|c|}
\hline \multicolumn{6}{|c|}{ EGFR-GITR-CD3 } \\
\hline $\begin{array}{c}\cos \\
\text { leoder }\end{array}$ & $\begin{array}{l}\text { EGFR } \\
\text { SCFv }\end{array}$ & \begin{tabular}{|c|} 
Cos \\
tinsee
\end{tabular} & $\begin{array}{c}\mathrm{cos} s \\
\mathrm{TM}\end{array}$ & GITR & $\mathrm{CD} 3 \zeta$ \\
\hline \multicolumn{6}{|c|}{ EGFR-AGITR-CD3 } \\
\hline $\begin{array}{c}\text { cos } \\
\text { lesder }\end{array}$ & $\begin{array}{l}\text { EGFR } \\
\text { SCFV }\end{array}$ & \begin{tabular}{|c|} 
cos \\
ningee
\end{tabular} & 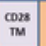 & $\Delta G I T R$ & $\mathrm{CD} \zeta$ \\
\hline \multicolumn{6}{|c|}{ EGFR-CD3-GITR } \\
\hline$\underset{\text { leaser }}{\text { leoser }}$ & $\begin{array}{l}\text { EGFR } \\
\text { SCFV }\end{array}$ & \begin{tabular}{|c|} 
cos \\
tinger
\end{tabular} & $\begin{array}{c}c \cos \\
\mathrm{TM}\end{array}$ & $\operatorname{CD} 3 \zeta$ & GITR \\
\hline
\end{tabular}

C

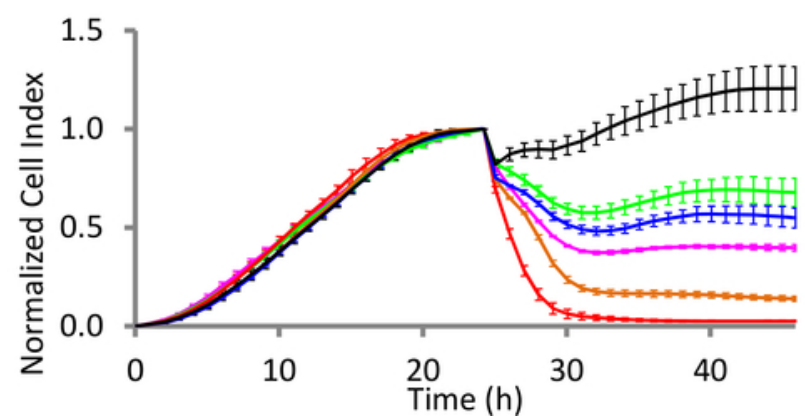

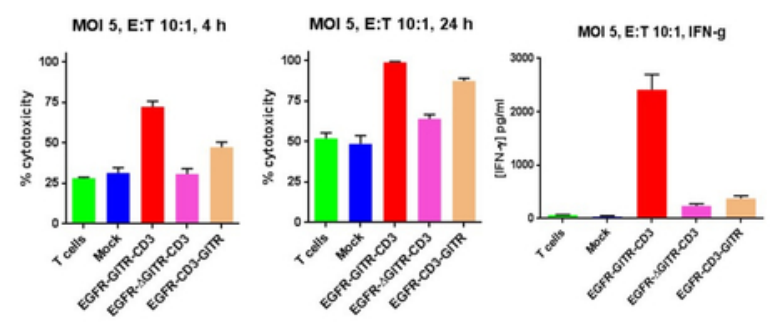

Figure 5: GITR domain increases CAR T cell cytotoxic activity against EGFR-positive tumor cell lines. (A) Different CAR constructs. (B) Bar plots of \% cytolysis for different CAR constructs at $4 \mathrm{~h}$ (left) and $24 \mathrm{~h}$ (middle). IFNy production at $24 \mathrm{~h}$ is shown on the right. (C) Continuous killing evaluation for all constructs. The black curve is the growth curve of BxPC3 ovarian cancer cells only. The green curve is the target cells treated with T cells only, the blue curve is the target cells treated with Mock CAR T cells, the red curve is the target cells treated with EGFRGITR-CD3-CAR T cells, the pink curve is the target cells treated with EGFR- $\triangle$ GITR-CD3-CAR T cells, and the brown curve is the target cells treated with EGFR-CD3-GITR-CAR T cells. Please click here to view a larger version of this figure.

\section{Discussion}

Chimeric antigen receptors are multidomain proteins composed of an extracellular single-chain variable fragment (scFv region), a hinge region, a transmembrane region, and a cytoplasmic domain composed of TCR signaling domains and additional costimulatory domains from receptors such as CD28 and OX40 11,40 . To design safe, selective, and efficacious CARs, it is imperative that the various permutations in the design of the CARs are thoroughly tested using in vitro potency assays and, eventually, animal models. In this study, we have provided a protocol and a workflow for how a real-time in vitro potency assay can inform the design of efficacious CARs.

In designing any type of potency assay, particularly for manufacturing purposes, it is imperative that the assay be sensitive, robust, consistent, and as close to the mechanism of action as possible ${ }^{16,17,41}$. The real-time potency assay described here is designed to measure the cytolytic activity of the CAR T cell directly rather than using a surrogate marker such as cytokine release. Importantly, the assay does not require any additional components such as dyes or reagents other than the assay plate (E-Plate) and the recommended media for maintaining the cells. Additionally, the assay is exquisitely sensitive and provides highly reproducible data compared to other label-based assays ${ }^{42,43,44}$. Moreover, the $x C E L L i g e n c e$ assay is amenable to using very low effector-to-target ratios which is ideal for the assessment of specific cytolysis.

In order to demonstrate the flexibility and utility of the xCELLigence system, we have focused on two tumor types, namely tumors of hematological origin and solid tumors. In order to assess the potency of CD22-directed CAR T cells, Raji cells (i.e., a B-cell lymphoma cell line) were tethered to the E-Plates using an anti-CD40 antibody. The tethering of Raji cells to the bottom of the E-Plates results in an impedance signal which reflects the viability and number of Raji cells in the well. Following the addition of CD22-CAR T cells, the Raji cells are selectively killed in a time- and effector-dependent manner, culminating in a time-dependent decrease in the impedance signal. The drop in impedance signifies cytolysis or loss of viability of the Raji cells ${ }^{17}$. This selective tethering approach using antibodies can be extended to other liquid tumor cell lines. An alternative strategy to using tumor cell lines of hematological origin is to use adherent cancer cells that are engineered to stably express the tumor antigens, such as CD19 expressed in HeLa cells. The advantage of this approach is that the parental HeLa cells are readily available and can be used as a negative control for specificity. Such an approach has already been validated with $\mathrm{CHO}-\mathrm{CD} 22$ vs. $\mathrm{CHO}$ cells and $\mathrm{CHO}-\mathrm{BCMAs}$ vs. $\mathrm{CHO}$ cells ${ }^{29}$. Using such different approaches, CAR T-cell design and efficacy can be easily tested. The xCELLigence assay is inherently flexible, and assay conditions can be adjusted in order to maximally approximate physiological conditions.

One major advantage of the potency assay we described here is that it is a simple functional assay and can be used in conjunction with genetic engineering techniques to design optimal and efficacious CARs in a high-throughput fashion. As was shown here for CAR T cells designed to target EGFR-positive cancer cells, the assay can be used to evaluate the relative activity of different CAR constructs/mutants. For example, we showed that when the GITR domain is located upstream of the CD3 domain it shows much more robust cytolytic activity than when it is located downstream of the CD3 domain.

Although it doesn't correlate perfectly with in vivo CAR T cell activity, in vitro cytokine release has historically been used as a measure of CAR T cell potency. Although the xCELLigence-based potency assay described here can be used for evaluating cell-based therapies during manufacturing or before being released for clinical application, how well these results correlate with in vivo efficacy has not yet been established. In vivo efficacy depends on a host of factors and variables which may not be recapitulated within an in vitro assay. Such variables include: homing of the CAR T cells to the site of the tumor, the stimulation and activation of the CAR T cell and its ability to persist within the patient, and 
the tumor microenvironment. With further refinement the xCELLigence assay may be capable of modeling some of these complex processes in vitro.

The protocol provided here applies to most adherent cancer cell lines and some of the liquid tumor cell lines. Clinical samples such as primary cancer cells, however, need to be further tested and optimized due to the complexity of the tumor types and phases. It is certainly worth noting that the in vitro potency assay system described here uses cancer cell lines only to reflect the potential activity of the CAR T cells. The real tumor situation inside the human body is much more complex, especially when a solid tumor is targeted, due to the dynamic tumor environment and development. Therefore, the potency evaluation result may not translate very well into the clinical efficacy of the CAR T cells tested.

In summary, the presented impedance-based xCELLigence platform allows label-free monitoring of cell killing for an extended period of time, namely up to 10 days. This capacity for such a long temporal scale for data collection differentiates the technology from other assays that are currently in use and which require setting up multiple experimental replicates for time point collection and laborious sample manipulation. Furthermore, the minimal signal contribution of the effector immune cells simplifies data analysis. The software can process data automatically and generate useful parameters such as the percentage of cytolysis, KT50, etc. The technology has already demonstrated high sensitivity (with E:T ratios as low as 1:20) and a large dynamic range (with E:T ratios from 20:1 to 1:20), which is not easily achieved with other assays. Overall, the implementation of this technology should allow a more accurate data analysis on a higher throughput scale that will enhance the development of CAR T-cell reagents, advancing the field at a much higher pace.

\section{Disclosures}

The authors conduct research in CAR T-cell development and associated potency assays which are business interests of ProMab Biotechnologies and ACEA Biosciences, respectively. However, this does not alter the authors' adherence to JoVE's mission of increasing the dissemination of scientific knowledge. The terms of this publication have been reviewed and approved by ProMab Biotechnologies and ACEA Biosciences in accordance with its policy of research.

\section{Acknowledgments}

The authors thank ProMab Biotechnologies and ACEA Biosciences for providing the reagents and instruments utilized in this study.

\section{References}

1. Miliotou, A. N., Papadopoulou, L. C. CAR T-cell Therapy: A New Era in Cancer Immunotherapy. Current Pharmaceutical Biotechnology. 19 (1), 5-18 (2018).

2. June, C. H., O'Connor, R. S., Kawalekar, O. U., Ghassemi, S., Milone, M. C. CAR T cell immunotherapy for human cancer. Science. 359 (6382), 1361-1365 (2018).

3. FDA. FDA approval brings first gene therapy to the United States. https://www.fda.gov/NewsEvents/Newsroom/PressAnnouncements/ ucm574058.htm (2017).

4. FDA. FDA approves CAR-T cell therapy to treat adults with certain types of large B-cell lymphoma. https://www.fda.gov/newsevents/ newsroom/pressannouncements/ucm581216.htm (2017).

5. Celgene. Celgene Corporation and bluebird bio Announce bb2121 Anti-BCMA CAR-T Cell Therapy Has Been Granted Breakthrough Therapy Designation from FDA and Prime Eligibility from EMA for Relapsed and Refractory Multiple Myeloma. http://ir.celgene.com/releasedetail.cfm? releaseid=1049014 (2017).

6. Liu, B., Song, Y., Liu, D. Clinical trials of CAR-T cells in China. Journal of Hematology \& Oncology. 10 (1), 166 (2017)

7. Fry, T. J. et al. CD22-targeted CAR T cells induce remission in B-ALL that is naive or resistant to CD19-targeted CAR immunotherapy. Nature Medicine. 24 (1), 20-28 (2018).

8. Yang, Y., Jacoby, E., Fry, T. J. Challenges and opportunities of allogeneic donor-derived CAR T cells. Current Opinion in Hematology. 22 (6), 509-515 (2015).

9. Celyad. Celyad Announces FDA Acceptance of IND Application for CYAD-101, a First-in-Class Non-Gene Edited Allogeneic CAR-T Candidate. https://www.celyad.com/en/news/celyad-announces-fda-acceptance-of-ind-application-for-cyad-101-a-first-in-class-non-geneedited-allogeneic-car-t-candidate (2017).

10. Sheridan, C. Allogene and Celularity move CAR-T therapy off the shelf. Nature Biotechnology. 36 (5), 375-377 (2018).

11. D'Aloia, M. M., Zizzari, I. G., Sacchetti, B., Pierelli, L., Alimandi, M. CAR-T cells: the long and winding road to solid tumors. Cell Death \& Disease. 9 (3), 282 (2018).

12. $\mathrm{Xu}$, J. et al. Combination therapy: A feasibility strategy for CAR-T cell therapy in the treatment of solid tumors. Oncology Letters. 16 (2), 2063-2070 (2018)

13. Xia, A. L., Wang, X. C., Lu, Y. J., Lu, X. J., Sun, B. Chimeric-antigen receptor T (CAR-T) cell therapy for solid tumors: challenges and opportunities. Oncotarget. 8 (52), 90521-90531 (2017).

14. Yong, C. S. M. et al. CAR T-cell therapy of solid tumors. Immunology and Cell Biology. 95 (4), 356-363 (2017).

15. Newick, K., O'Brien, S., Moon, E., Albelda, S. M. CAR T Cell Therapy for Solid Tumors. Annual Review of Medicine. 68, 139-152 (2017).

16. de Wolf, C., van de Bovenkamp, M., Hoefnagel, M. Regulatory perspective on in vitro potency assays for human mesenchymal stromal cells used in immunotherapy. Cytotherapy. 19 (7), 784-797 (2017).

17. Cerignoli, F. et al. In vitro immunotherapy potency assays using real-time cell analysis. PLOS ONE. (2018).

18. Holden, H. T., Oldham, R. K., Ortaldo, J. R., Herberman, R. B. Standardization of the chromium- 51 release, cell-mediated cytotoxicity assay: cryopreservation of mouse effector and target cells. Journal of the National Cancer Institute. 58 (3), 611-622 (1977).

19. Somanchi, S. S., McCulley, K. J., Somanchi, A., Chan, L. L., Lee, D. A. A Novel Method for Assessment of Natural Killer Cell Cytotoxicity Using Image Cytometry. PLOS ONE. 10 (10), e0141074 (2015). 
20. Mukherjee, M., Mace, E. M., Carisey, A. F., Ahmed, N., Orange, J. S. Quantitative Imaging Approaches to Study the CAR Immunological Synapse. Molecular Therapy: The Journal of the American Society of Gene Therapy. 25 (8), 1757-1768 (2017).

21. Zaritskaya, L., Shurin, M. R., Sayers, T. J., Malyguine, A. M. New flow cytometric assays for monitoring cell-mediated cytotoxicity. Expert Review of Vaccines. 9 (6), 601-616 (2010).

22. Nelson, D. L., Kurman, C. C., Serbousek, D. E. Chapter 7, Unit 7: 51Cr release assay of antibody-dependent cell-mediated cytotoxicity (ADCC). Current Protocols in Immunology. 27 (2001).

23. Abassi, Y. A. et al. Label-free, real-time monitoring of IgE-mediated mast cell activation on microelectronic cell sensor arrays. Journal of Immunological Methods. 292 (1-2), 195-205 (2004).

24. Glamann, J., Hansen, A. J. Dynamic detection of natural killer cell-mediated cytotoxicity and cell adhesion by electrical impedance measurements. Assay and Drug Development Technologies. 4 (5), 555-563 (2006).

25. Solly, K., Wang, X., Xu, X., Strulovici, B., Zheng, W. Application of real-time cell electronic sensing (RT-CES) technology to cell-based assays Assay and Drug Development Technologies. 2 (4), 363-372 (2004).

26. Zhu, J., Wang, X., Xu, X., Abassi, Y. A. Dynamic and label-free monitoring of natural killer cell cytotoxic activity using electronic cell sensor arrays. Journal of Immunological Methods. 309 (1-2), 25-33 (2006).

27. Ke, N., Wang, X., Xu, X., Abassi, Y. A. The xCELLigence system for real-time and label-free monitoring of cell viability. Methods in Molecular Biology. 740, 33-43 (2011).

28. Lamarche, B. J., Xi, B., Cerignoli, F. Quantifying the Potency of Cancer Immunotherapies: Immune Cell-Mediated Killing Kinetics and Efficacy Analysis in Real-Time without the Use of Labels. Genetic Engineering \& Biotechnology News (GEN). 36 (14), 18-19 (2016).

29. Golubovskaya, V. et al. CD47-CAR-T Cells Effectively Kill Target Cancer Cells and Block Pancreatic Tumor Growth. Cancers. 9 (10) (2017).

30. Golubovskaya, V. M. et al. GITR domain inside CAR co-stimulates activity of CAR-T cells against cancer. Frontiers in Bioscience. 23, 2245-2254 (2018).

31. Guedan, S. et al. Enhancing CAR T cell persistence through ICOS and 4-1BB costimulation. JCI Insight. 3 (1) (2018).

32. Erskine, C. L., Henle, A. M., Knutson, K. L. Determining optimal cytotoxic activity of human Her2neu specific CD8 T cells by comparing the Cr51 release assay to the xCELLigence system. Journal of Visualized Experiments. (66), e3683 (2012).

33. Davenport, A. J. et al. CAR-T Cells Inflict Sequential Killing of Multiple Tumor Target Cells. Cancer Immunology Research. 3 (5), $483-494$ (2015).

34. Hegde, M. et al. Tandem CAR T cells targeting HER2 and IL13Ralpha2 mitigate tumor antigen escape. The Journal of Clinical Investigation. 126 (8), 3036-3052 (2016).

35. Jin, J. et al. Enhanced clinical-scale manufacturing of TCR transduced T-cells using closed culture system modules. Journal of Translational Medicine. 16 (1), 13 (2018).

36. Weiskopf, K. Cancer immunotherapy targeting the CD47/SIRPalpha axis. European Journal of Cancer. 76, 100-109 (2017).

37. Huang, Y., Ma, Y., Gao, P., Yao, Z. Targeting CD47: the achievements and concerns of current studies on cancer immunotherapy. Journal of Thoracic Disease. 9 (2), E168-E174 (2017).

38. Ma, S., Thorpe, P., Vitetta, E., Meyer, J. Combined targeting of exposed phosphatidylserine, CD47 and CD54 on human pancreatic tumor cells in a mouse xenograft model of human pancreatic cancer (P4455). The Journal of Immunology. 190 (1 Supplement) (2013).

39. Yamamoto, K. et al. Emergence of CD47- high expression cells confers enhanced tumorigenicity upon KDM6B suppression in pancreatic cancer. Cancer Research. 76 (2 Supplement) (2016).

40. Xu, D. et al. The development of CAR design for tumor CAR-T cell therapy. Oncotarget. 9 (17), 13991-14004 (2018).

41. FDA. Guidance for Industry, Potency Tests for Cellular and Gene Therapy Products. https://www.fda.gov/BiologicsBloodVaccines/ GuidanceComplianceRegulatoryInformation/Guidances/CellularandGeneTherapy/ucm072571.htm (2017).

42. Limame, R. et al. Comparative analysis of dynamic cell viability, migration and invasion assessments by novel real-time technology and classic endpoint assays. PLOS ONE. 7 (10), e46536 (2012).

43. Chiu, C. H. et al. Comparison between xCELLigence biosensor technology and conventional cell culture system for real-time monitoring human tenocytes proliferation and drugs cytotoxicity screening. Journal of Orthopaedic Surgery and Research. 12 (1), 149 (2017).

44. Hillger, J. M., Lieuw, W. L., Heitman, L. H., IJzerman, A.P. Label-free technology and patient cells: from early drug development to precision medicine. Drug Discovery Today. 22 (12), 1808-1815 (2017). 